\title{
Switched parasitic antennas and controlled reactance parasitic antennas: A systems comparison.
}

\author{
David V. Thiel, Radio Science Laboratory, \\ Griffith University, Nathan Qld Australia. d.thiel@griffith.edu.au
}

\section{Introduction}

In 1978 Harrington [1] proposed a dipole array consisting of a center fed dipole surrounded by a ring of parasitic dipoles. Each parasitic element contained a reactive impedance element at the center. By controlling each reactance independently, the principal radiation direction could be varied through $360^{\circ}$ in the azimuthal plane. A simplification of the idea replaced the variable reactance loads with on/off switches [2]. A number of researchers have followed up this work with configurations of monopoles and the ability to control the principal direction of radiation has been verified [3-6]. The size of the monopole ground plane can be made quite small if a skirt is used $[7,8]$. Switched parasitic antennas have since been designed for simultaneous dual band operation, multi-beam operation and direction finding [6].

These antenna configurations have a number of advantages over phased arrays and active integrated antennas. If the configuration of the parasitic elements remains symmetrical about the plane of the principal beam direction, then the input impedance of the antenna is independent of beam direction. The construction of the antennas is relatively inexpensive as no phase shifters or wave splitters are required. The antenna footprint is relatively small as the arrays rely on significant mutual coupling and so the inter-element spacing is usually less than 0.5 wavelengths whereas in phased arrays the element spacing must be larger to minimize scan blindness caused by mutual coupling.

The two antenna arrays (i.e. variable reactance and switched parasitic antennas) form part of a larger number of antenna types targeted for use as smart antennas (or adaptive antennas). Some of the more promising applications of smart antennas include direction finding, self-assembling wireless networks, and tracking in mobile communications systems. In this paper the systems requirements of these applications are discussed in the light of the performance characteristics of these two antenna types. In all examples given we will assume that the antenna is in the receiving mode.

\section{Theory}

2.1 Beam width. The antenna gain and the minimum possible beam width of any antenna is determined by the size of the antenna. For an array of monopoles, this dimension is the diameter of the parasitic ring. Experimentally verified gain from a number of $\mathrm{GA}$ optimised antenna configurations increases from $6.3 \mathrm{dBi}$ to 
$9 \mathrm{dBi}$ accompanied by a decrease in beam width from $130^{\circ}$ to $60^{\circ}$ for $n=1$ to 6 where $n$ is the number of parasitic elements in a single ring. The ring radius varied from $0.13 \lambda_{0}$ to $0.3 \lambda_{0}$ for these optimised arrays.

To obtain total coverage, it is desirable that the intersection between adjacent switched beams is less than $3 \mathrm{~dB}$ down on the main beam gain. In the case of the reactively controlled antenna, this is possible if there are sufficient steps in the control voltage for the varactors. In the case of the switched parasitic antenna, this is possible if the antenna has been optimized.

Thus, if the $3 \mathrm{~dB}$ beam width of the array is $B$ then it is desirable that $n B<360^{\circ}$ for maximum azimuthal field coverage. If $B<360 / n$, then there is a possibility that some targets may not be detected.

2.2 Switches and varactors. The performance of both antennas is dependent on the characteristics of the lumped element at the feed point of the parasitic elements. In the case of switched parasitic antennas, the open circuit capacitance must present a reactance of magnitude greater than $1.2 \mathrm{k} \Omega$ and the short circuit less than $800 \Omega[5,6]$. Providing all switches in the array conform to these specifications at the frequency of interest, the antenna will perform satisfactorily. If p.i.n. diode switches are used, operating frequencies of less than $2 \mathrm{GHz}$ usually ensure reliable results. The use of RF MEMs switches can greatly increase the frequency range of operation.

In the case of varactors, the capacitance $C$ is controlled by an applied voltage $V$. As the performance of these devices is continuous, then the uniformity of both the applied voltage and the $C(V)$ are critical to the performance of the antenna. For optimised performance, the reactance must vary from positive (inductive) to negative (capacitive) and this has been achieved using a transmission line/varactor combination [8].

Small manufacturing errors in the varactors, their transmission lines, mounting pads or the DC voltages applied can lead to errors in alignment of the main beam and changes in the input impedance of the antenna.

2.3 Computer support. Switched parasitic antennas require one dedicated digital line for each parasitic element. No further signal conditioning or components are required between the microcontroller and the switch for p.i.n. diodes. In receiving mode, the control loop includes an RF detector providing an analog input to a microcontroller and $n$ digital outputs controlling each of the switches in the parasitic elements. The switches used are open circuit when the applied voltage is zero. The current drain is therefore that required for the $n-1$ short circuited switches and the microcontroller.

In the case of the varactor antenna, $n$ digital to analog converter (DAC) circuits are required to convert the digital microcontroller output to a voltage level. In 
addition, a stable reference voltage must be provided to each of the DAC circuits. A defined voltage must be maintained on all varactors at all times. The current drain on the system is therefore the sum of that required by the $n$ DAC circuits and $n$ varactors in addition to the microcontroller. The microcontroller also needs to have a relatively large number of digital output ports to service the DACs.

\section{Applications}

3.1 Tracking moving transmitters/ Direction finding. The automatic location and tracking of a moving transmitter requires a number of different computer controlled processes. Initially the search for a target(s) requires a complete scan of all possible beam positions. The time required to do this depends on the number of possible beam positions, the time taken to identify the signal as being relevant and the time it takes to reposition the main beam of the antenna.

The switched parasitic antenna has $n$ possible main beam positions and so scanning can be relatively quick. The precise angular determination of the transmitter relative to the antenna can be determined by a relatively simple mathematical algorithm if only one transmitter is present and there is no multipath effect resulting in angular uncertainty [8].

The varactor controller array has a larger number of main beam positions which takes longer to scan. With a suitable DSP algorithm, the angular position of the source can be determined as well as the effect of multipath. However, in this application, considerable processing power is required. This increases the antenna response time unless the calculations are compled off line.

If the transmitter is moving, a global scan is required to update the precise position. After several global scans is may be possible to predict the path of the transmitter and use a dither technique to update the main beam direction [6]. If the system requirement is target location, then this must be done almost continually. If the system requirement is to keep a communications channel open to the moving transmitter, then the requirement to update the position is not so time critical with these antenna systems because of their relatively large beam widths. In the latter case, precise main beam positioning is not so critical.

3.2 Interference cancellation. One effective method of reducing the impact of an adjacent transmitter operating in the same band is to position a null in the radiation pattern in the direction of the interfering signal. If the angular separation between the interference and the desired channel is greater than approximately $45^{\circ}$, then for relatively small antenna systems, there will be sufficient gain in the remaining $270^{\circ}$ to ensure reliable reception. The accurate positioning of the null in such a way that the field strength of the interfering signal is reduced relative to the desired signal to achieve an improvement in the signal to interfering/noise ratio (SINR) of $15 \mathrm{~dB}$ (say) can only be achieved if accurate null position is possible. The precision required might be as high as $2^{\circ}$ if the radiation nulls are very sharply defined. 
A switched parasitic antenna system may not be capable of achieving this level of precision. The reactively controlled parasitic anterna however can achieve this control provided there is sufficient precision in the varactor voltages derived from the microcontroller. The positioning of the null requires an optimization routine similar to the direction finding routine discussed in Section 3.1. Note that imperfect control of the varactors and variation in the over-all construction of the antenna can result in a poor quality null in the main radiation beam.

\section{Conclusions}

A comparison has been presented between the characteristics of small switched parasitic antenna arrays and varactor controlled parasitic antenna arrays. As the beam width of both antennas is similar, it is concluded that the control system and support electronics is much simpler for the switched parasitic antennas. In most applications of these smart antennas, precise angular control of the main beam is not a critical issues for most applications. If null positioning or if precise direction finding of multiple sources is a major requirement, only the varactor controlled parasitic antenna type offers this functionality.

\section{References:}

[1] R.F. Harrington, "Reactively controlled directive arrays," IEEE Trans. Antennas and Propagation, vol. 26, pp. 390-395, 1978.

[2] R.M. Milne, "A small adaptive antenna for mobile communications," IEEE APS Symposium, pp. 797-800, 1985.

[3] T. Ohira and K. Gyoda, "Handheld microwave direction-of-arrival finder based on varactor-tuned aerial beamforming," Proc. Asia Pacific Microwave Conf. Sydney, pp. 585-588, 2001.

[4] A. Sibille, C. Roblin, and G. Poncelet, "Circular switched monopole arrays for beam steering wireless communications," Electronics Letters, vol. 33, pp. 551-

\section{2,1997}

[5] D.V. Thiel, S.G. O'Keefe and J.W. Lu, "Electronic beam steering in wire and patch systems using switched parasitic elements," IEEE APS Symposium, Seattle, pp. $534-537,1996$.

[6] D.V. Thiel and S. Smith, Switched parasitic antennas for cellular communications, Artech House, 2002.

[7] D.V. Thiel, "Tin-can antenna - a switched parasitic monopole antenna on a finite ground plane with a conductive sleeve," $7^{\text {th }}$ Australia Symp. Antennas, Sydney. p. 19, 2001

[8] R. Schlub, J. Lu, and T. Ohira, "Seven-element ground skirt monopole ESPAR antenna design from a genetic algorithm and finite element method," IEEE Trans. Antennas and Propagation, vol. 51, pp. 3033-3039, 2003.

[8] S. L. Preston, D.V. Thiel, T. A. Smith, S.G. O'Keefe and J.W. Lu, "Base station tracking in mobile communications using switched parasitic antenna array," IEEE Trans. Antennas and Propagation, vol. 46, pp. 841-844, 1998. 